\title{
Outbreaks of Highly Pathogenic Avian Influenza (H5N6) Virus Subclade 2.3.4.4h in Swans, Xinjiang, Western China, 2020
}

Yanbing Li, Minghui Li, Yulei Li, Jingman Tian, Xiaoli Bai, Cen Yang, Jianzhong Shi, Ridengcaicike Ai, Weidong Chen, Wentao Zhang, Jie Li, Yufei Kong, Yuntao Guan, Hualan Chen

In January 2020 , the subclade $2.3 .4 .4 \mathrm{~h}$ of highly pathogenic avian influenza (H5N6) virus infected migratory whooper swans and mute swans in Xinjiang, western China. The virus is lethal to chickens and ducks but has low pathogenicity in mice. Antigenically, this subclade is similar to the H5N1 vaccine seed virus Re-11.

$\mathrm{T}$ he H5 highly pathogenic avian influenza viruses (HPAIVs) of clade 2.3.4.4 are of great concern because of their global spread and circulation. Ample evidence indicates that clade 2.3.4.4 H5 viruses derived neuraminidase (NA) gene from other lowpathogenicity avian influenza viruses (LPAIVs) cocirculating in migratory birds, and new subtypes of H5N2, H5N5, H5N6, and H5N8 HPAIVs have been detected in wild bird species and poultry globally $(1,2)$. To date, $\mathrm{H} 5$ viruses of clade 2.3.4.4 have evolved into 8 subclades (2.3.4.4a to 2.3.4.4h) according to the World Health Organization's (WHO) nomenclature system (1). Among them, H5N6 is the only subtype that has caused human infections. As of August 2019, a total of 24 human cases have been reported to $\mathrm{WHO}$; the mortality rate is $67 \%(3,4)$.

H5N6 virus of subclade 2.3.4.4a was first detected in poultry in Laos in 2013, then spread to Vietnam and China and caused numerous cases in these ar-

Author affiliations: State Key Laboratory of Veterinary Biotechnology, Harbin Veterinary Research Institute, Chinese Academy of

Agricultural Sciences, Harbin, Heilongjiang, China (Yanbing Li, M. Li, Yulei Li, J. Tian, X. Bai, J. Shi, C. Yang, Y. Kong, Y. Guan, H. Chen); Preventive and Control Center for Animal Disease of Xinjiang Uyghur Autonomous Region, Urumqi, Xinjiang Wei Autonomous Region, China (R. Ai, J. Li); Preventive and Control Center for Animal Disease of Xinjiang Crops, Urumqi (W. Zhang, J. Li)

DOI: https://doi.org/10.3201/eid2612.201201 eas. $\mathrm{H} 5 \mathrm{~N} 8$ virus of subclade 2.3.4.4b caused disease outbreaks in wild birds and poultry in Korea in 2014, then spread to North America through bird migration and established a new subclade, 2.3.2.4c. When the $\mathrm{H} 5 \mathrm{~N} 8$ virus of subclade 2.3.4.4b landed in Europe and Africa, it reassorted with the local LPAIV and produced H5N6 with a novel internal gene cassette in 2017 (5). Simultaneously, the H5N6 viruses of subclades 2.3.4.4d, 2.3.4.4e, 2.3.4.4f, 2.3.4.4g, and 2.3.4.4h established in poultry and wild birds in Southeast Asia (1,6-8). Among the 8 subclades of 2.3.4.4, only 3 (H5N6 2.3.4.4b, 2.3.4.4e, and 2.3.4.4f) had been previously detected in swans (1).

Since 2004, different vaccines have been developed and widely administered among poultry flocks in China and other countries for H5 avian influenza control, and the vaccine seed viruses used in China have been updated regularly to ensure antigenic match between the vaccine strain and the prevalent strains $(9,10)$. After the H7N9 HPAIVs emerged in China in 2017, an H5/H7 combined inactivated vaccine was developed and used in poultry $(11,12)$. Currently, the vaccine seed virus Re-11 is being used to control the clade 2.3.4.4 viruses (10). In our study, we analyzed the genetic evolution, antigenicity, and pathogenicity of the H5N6 HPAIVs isolated from migratory whooper swans (Cygnus cygnus) and mute swans (C. olor) in Xinjiang, western China, in January 2020.

\section{The Study}

The first sick whooper swan was found on December 29, 2019, in Sala Village, Samuyuzi Township, Yining City, Xinjiang Uyghur Autonomous Region. The bird died on January 1, 2020. By January 17, deaths had been reported in 58 swans in 6 locations (Table 1; Figure 1, panel A). We received 5 batches of clinical 
Table 1. Avian influenza (H5N6) outbreaks among migratory whooper swans (Cygnus cygnus) and mute swans (C. olor), Xinjiang Province, China, January 2020

\begin{tabular}{|c|c|c|c|c|}
\hline \multirow[b]{3}{*}{ Time } & \multirow[b]{3}{*}{ Location description } & \multicolumn{3}{|c|}{ Bird information } \\
\hline & & \multirow[b]{2}{*}{ Flock size* } & \multicolumn{2}{|c|}{ No. swans† } \\
\hline & & & Total & Dead \\
\hline 2019 Dec 29 to 2020 Jan 5 & Small lake in Yining County, Ili Kazak City & $>100$ & 100 & 10 \\
\hline 2020 Jan $1-6$ & Natural park in Yining County, Ili Kazak City & $>2,300$ & $40(270)$ & $6(3)$ \\
\hline 2020 Jan $1-8$ & Natural park in Bole County, Botorla City & 160 & 55 & 6 \\
\hline 2020 Jan $8-10$ & Natural park in Hejing County, Bayingola City & 1150 & 150 & 1 \\
\hline 2020 Jan $12-14$ & Wetland in Manas County, Changji City & 2,000 & 800 & 13 \\
\hline 2020 Jan $17-20$ & Water reservoir in Maguan Chu County, Shihezi City & 1,000 & 150 & 19 \\
\hline
\end{tabular}
†Numbers are whooper swans, except numbers in parentheses, which are mute swans.

samples from 13 dead birds (11 whooper swans and 2 mute swans), and 13 H5N6 viruses were isolated. The hemagglutinin (HA) subtypes were identified by a hemagglutinin-inhibition test with a panel of H1H16 subtype antisera, whereas the NA subtypes were detected by reverse transcription PCR with a panel of N1-N9 subtype-specific primers (11).

To trace the origin of the viruses and understand their genetic relationship, we sequenced the genome of the 13 viruses and performed comparative phylogenetic analysis with the representative H5 HPAIVs that were recommended by WHO (1). All 13 H5N6 viruses possess high identity with each other $(99.5 \%-$ $100 \%) ; 7$ of 8 segments are closely related to the H5N6 virus isolated from environmental samples in Guangdong Province in 2017, whereas the other 1, nonstruc- tural protein gene, is closest to $\mathrm{A} /$ chicken/Nghe An/01VTC/2018(H5N6) (Appendix Table 1, https:/ / wwwnc.cdc.gov/EID/article/26/12/20-1201-App1. pdf). The HA gene has the typical highly pathogenic amino acid sequence -RRKR-in its cleavage site, and a few mammalian adaptation mutations were detected in the genome (Table 2) (13). In the maximum clade credibility tree, the HA genes of the $13 \mathrm{H} 5 \mathrm{~N} 6$ viruses are grouped into subclade 2.3.4.4h with the HA genes of the strains recently found in Vietnam, China, and Russia (1) (Figure 1, panel B). The neighbor-joining phylogenic trees of the 8 gene segments are shown in Appendix Figure 1.

The hemagglutinin-inhibition test was performed with polyclonal antiserum generated from the SW/ $\mathrm{XJ} / 1 / 2020(\mathrm{H} 5 \mathrm{~N} 6)$ and the currently used H5N1

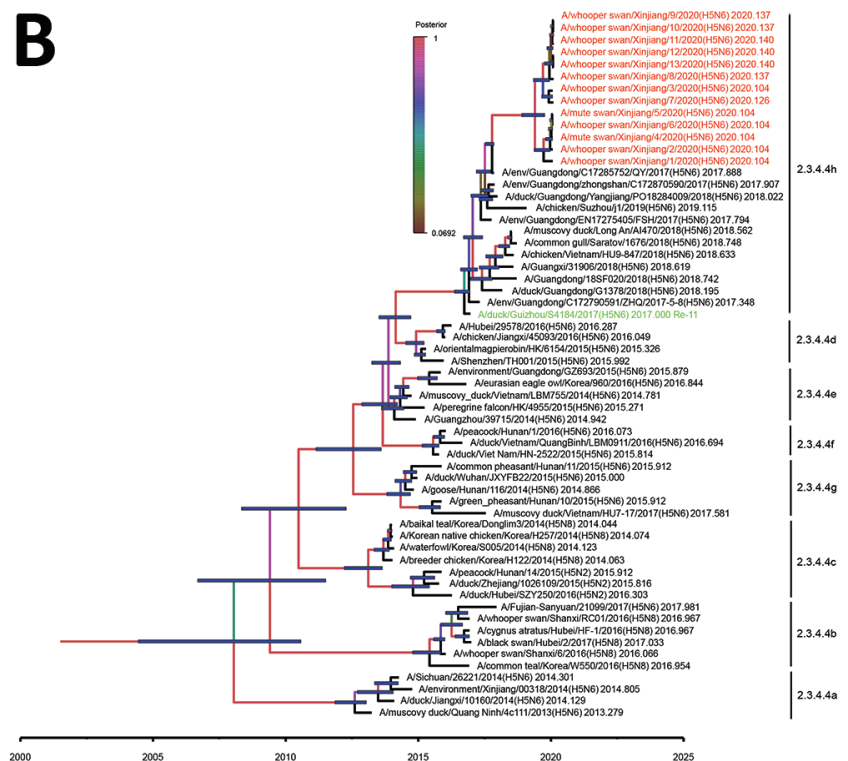

B

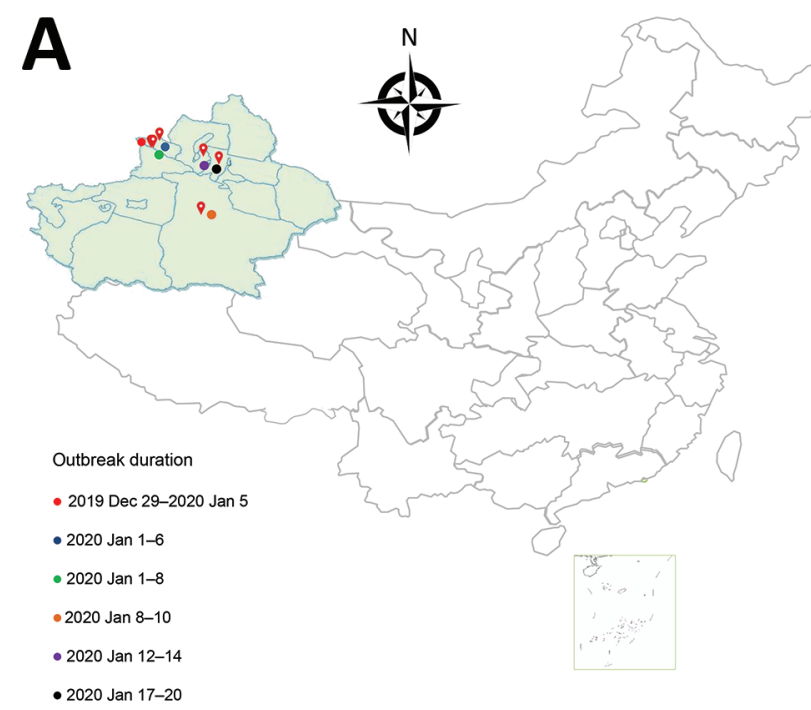
Figure 1. Geography and phylogeny of avian influenza (H5N6) outbreaks among migratory whooper swans (Cygnus cygnus) and
mute swans (C. olor), Xinjiang Province, China, January 2020. A) Disease outbreak sites are marked with red drops, and dates of the outbreaks are indicated. Inset map shows islands in the South China Sea. B) Phylogenetic tree of the hemagglutinin (HA) genes of H5 viruses. The HA gene maximum clade credibility tree of the H5 viruses was constructed by using the BEAST 1.8 .4 software package (https://beast-dev.github.io/beast-mcmc). Node bars indicate $95 \%$ highest posterior density of the node height. Each branch is colored by posterior probability: the $13 \mathrm{H} 5 \mathrm{~N} 6$ viruses reported in this study are shown in red and the HA donor of the H5N1 vaccine Re-11 in green. The time to the most recent common ancestor is labeled at the bottom of the tree, which was estimated by using the Bayesian Markov chain Monte Carlo method in the BEAST 1.8.4 software package. 
Table 2. Virulence related molecular markers detected in the $\mathrm{WS} / \mathrm{XJ} / 1 / 2020$ (H5N6) virus detected among migratory whooper swans (Cygnus cygnus) and mute swans (C. olor), Xinjiang Province, China, January 2020

\begin{tabular}{lcc}
\hline Protein & Amino acid/motif & Phenotypic consequences \\
\hline Hemagglutinin & Cleavage site motif: -RRKR ${ }^{-}$G- & $\begin{array}{c}\text { Polybasic cleavage motif sequence required for high pathogenicity } \\
\text { of avian influenza viruses in chickens }\end{array}$ \\
\hline Neuraminidase & Stalk deletion 58-68 & Increased virulence in mice \\
\hline $\begin{array}{l}\text { Polymerase acidic } \\
\text { protein }\end{array}$ & $515 \mathrm{~T}$ & Increased polymerase activity in mammalian cells \\
\hline Matrix protein 1 & $30 \mathrm{D}$ & Increased virulence in mice \\
& $215 \mathrm{~A}$ & Increased virulence in mice \\
\hline Nonstructural & $80-84$ deletion & Increased virulence in mice \\
protein 1 & $42 \mathrm{~S}$ & Increased virulence in mice \\
& $98 \mathrm{~F}$ & Increased virulence in mice \\
& $101 \mathrm{M}$ & Increased virulence in mice \\
Increased virulence in mice
\end{tabular}

inactivated vaccine Re-11, which carries the HA gene from A/duck/Guizhou/S4184/2017(H5N6) virus (10). We found that the SW/XJ/1/2020(H5N6) cross-reacted well with Re-11 antisera, and vice versa (Appendix Table 2), yielding a cross-reactivity $R$ value of 0.26 .

We conducted an intravenous pathogenicity index test in chickens with the index virus, WS/ XJ/1/2020(H5N6), by following the protocol of the World Organisation for Animal Health (OIE) (14). Ten 6-week-old specific-pathogen-free chickens were inoculated with $0.2 \mathrm{~mL}$ of virus intravenously, and all the birds died within 3 days postinoculation, yielding an intravenous pathogenicity index test value of 2.59 .
We tested the virulence and transmission of the WS/XJ/1/2020(H5N6) in ducks as previously described (2). Eight 3-week-old specific-pathogen-free ducks were intranasally inoculated with $10^{6} 50 \%$ egg infective dose $\left(\mathrm{EID}_{50}\right) \mathrm{WS} / \mathrm{XJ} / 1 / 2020(\mathrm{H} 5 \mathrm{~N} 6)$, and 3 uninfected ducks were put in the same cage 24 hours later for monitoring transmission. Three virus-inoculated ducks were euthanized on day 3 postinoculation, and high titers of virus were detected in the tested organs (Figure 2, panel A). Virus was also detected in the oropharyngeal and cloacal swabs of the surviving virus-inoculated ducks and the contact ducks on days 3 and 5 postinoculation (Figure 2, panel B). All
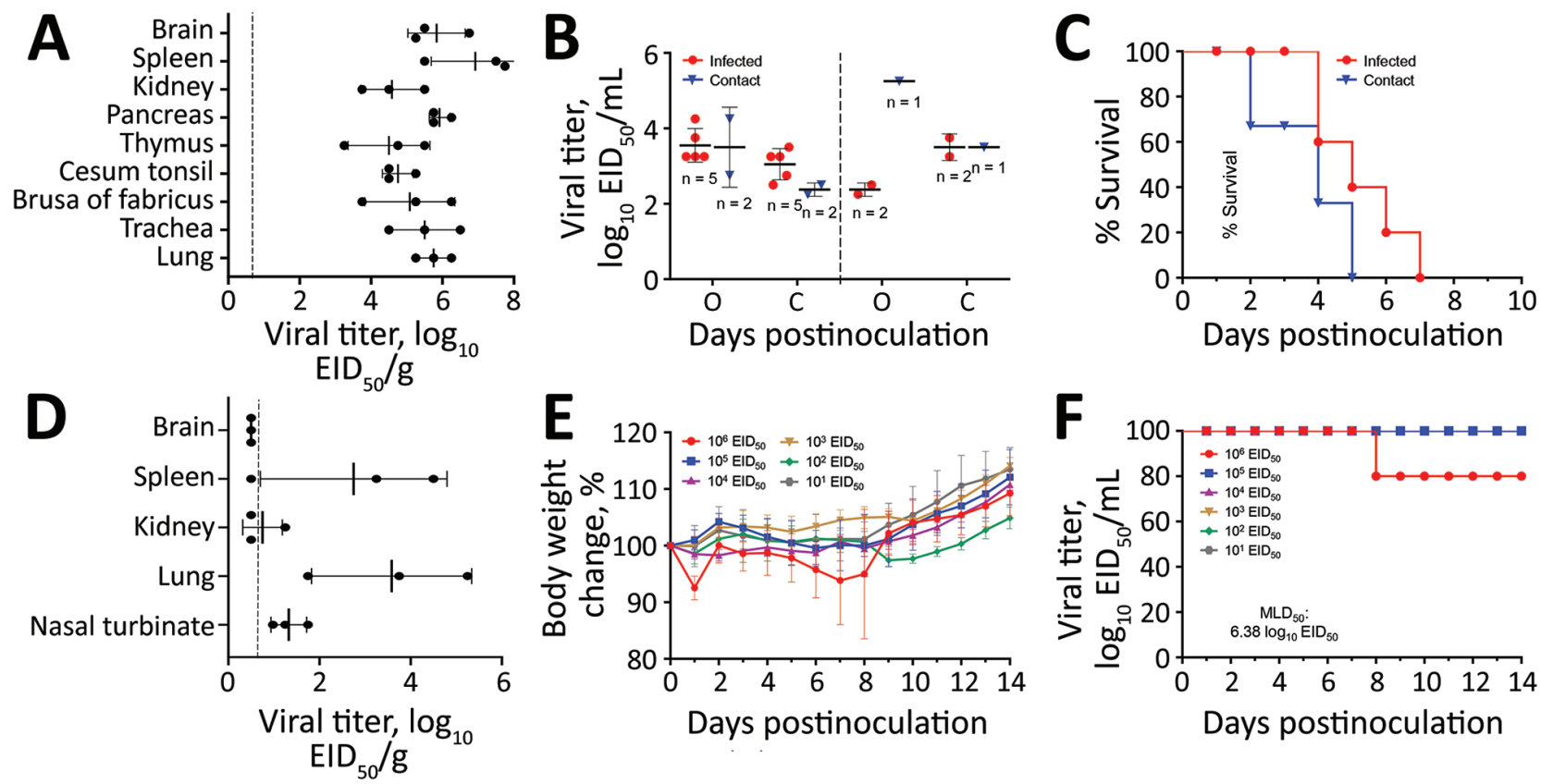

Figure 2. Replication and virulence of the $\mathrm{WS} / \mathrm{XJ} / 1 / 2020(\mathrm{H} 5 \mathrm{~N} 6)$ isolate in ducks and mice in a laboratory test performed after H5N6 avian influenza (H5N6) outbreaks among migratory whooper swans (Cygnus cygnus), Xinjiang Province, China, January 2020. A) Viral titer in organs of ducks that were euthanized on day 3 postinoculation. B) Viral titers in oropharyngeal and cloacal swabs from all surviving ducks were collected on days 3 and 5 postinoculation. C) Lethality of the virus in ducks. D) Viral titer in organs of mice that were euthanized on day 3 postinoculation. E) Bodyweight change of mice after inoculation with different doses of the virus. $F$ ) MLD $_{50}$ of the virus. Viral titers in panels $A, B$, and $D$ are shown as the mean \pm SD. The dashed lines indicate the lower limit of detection. EID ${ }_{50}$, $50 \%$ egg infective dose; $M_{L} D_{50}, 50 \%$ mouse lethal dose. 
5 virus-inoculated ducks and 3 contact ducks died within 7 days postinoculation (Figure 2, panel C).

The replication and 50\% mouse lethal dose $\left(\mathrm{MLD}_{50}\right)$ of the WS/XJ/1/2020(H5N6) were evaluated in BALB/c mice as previously reported (2). Three mice were intranasally inoculated with $10^{6} \mathrm{EID}_{50}$ of WS/XJ/1/2020(H5N6) in a volume of $50 \mu \mathrm{L}$ and were euthanized on day 3 postinoculation to assess virus replication in organs, and we found the virus in the brain of 1 mouse, the spleens of 2 mice, and the nasal turbinates and lungs of all 3 mice, but not in the kidneys of any mouse (Figure 2, panel D). To test the $\mathrm{MLD}_{50^{\prime}}$ groups of five 6-week-old mice were intranasally inoculated with $10^{1}$ to $10^{6} \mathrm{EID}_{50}$ of WS/ $\mathrm{XJ} / 1 / 2020$ (H5N6) in a volume of $50 \mu \mathrm{L}$ and were monitored for bodyweight loss and death for 14 days. Only 1 of 5 mice that received the highest dose of $10^{6}$ EID $_{50}$ died on day 8 postinoculation; all other mice survived the 14-day observation period, yielding an $\mathrm{MLD}_{50}$ value of $6.38 \log _{10}$ EID $_{50}$ (Figure 2, panel E, F).

\section{Conclusions}

A total of 58 swans died from H5N6 virus infection in 6 wild bird habitats in Xinjiang in January 2020, and we isolated 13 similar H5N6 HPAIVs from the swan specimens. These viruses bear the HAs of subclade 2.3.4.4h, which were previously detected in other bird species but not in swans.

The WS/XJ/1/2020(H5N6) is highly pathogenic to chickens and ducks, and antigenically close to the $\mathrm{H} 5 \mathrm{~N} 1$ vaccine seed virus Re- 11 . Although the virus is low pathogenic in mice, it bears multiple residues that can increase its virulence in mammals, and thus might pose a potential threat to public health.

Wild birds carry and spread the H5 HPAIV, as evidenced by the dissemination of the clade 2.2 viruses from Asia to Europe and Africa in 2005, and the intercontinental distribution of the clade 2.3.4.4b viruses in $2014(5,15)$. The prospect of these H5N6 viruses detected in swans being distributed widely by wild birds is worrisome. Therefore, with the migratory season coming, surveillance and preventive measures should be implemented in poultry raised on the migration routes of wild birds.

\section{Acknowledgments}

We thank the researchers who submitted H5N6 HPAIVs sequences to GISAID. The sequence data from this study were deposited in GISAID with the accession numbers EPI1718935-9038.

This study was supported by the National Key R\&D Program of China (grant no. 2016YFD0500201), the National Natural Science Foundation of China (grant no.
31521005), the Natural Science Foundation of the Heilongjiang Province of China (grant no. ZD2018007), the Central Public-interest Scientific Institution Basal Research Fund for Chinese Academy of Agricultural Sciences (grant no. 1610302017001), and the China Agriculture Research System (grant no. CARS-41-G12).

\section{About the Author}

Dr. Li is a veterinary epidemiologist and virologist at Harbin Veterinary Research Institute, Chinese Academy of Agricultural Sciences, China. Her research focuses on the molecular epidemiology and pathogenicity of avian influenza viruses.

\section{References}

1. World Health Organization. Antigenic and genetic characteristics of zoonotic influenza A viruses and development of candidate vaccine viruses for pandemic preparedness. 2020 [cited 2020 Mar 10]. https:/ / www.who. int/influenza/vaccines/virus/202002_zoonotic_vaccine virusupdate.pdf

2. Cui Y, Li Y, Li M, Zhao L, Wang D, Tian J, et al. Evolution and extensive reassortment of $\mathrm{H} 5$ influenza viruses isolated from wild birds in China over the past decade. Emerg Microbes Infect. 2020;9:1793-803. https:/ / doi.org/10.1080/ 22221751.2020.1797542

3. Yang L, Zhao X, Li X, Bo H, Li D, Liu J, et al. Case report for human infection with a highly pathogenic avian influenza A(H5N6) virus in Beijing, China 2019. Biosafety and Health. 2020;2:49-52. https:// doi.org/10.1016/j.bsheal.2020.02.003

4. Yang L, Zhu W, Li X, Bo H, Zhang Y, Zou S, et al. Genesis and dissemination of highly pathogenic H5N6 avian influenza viruses. J Virol. 2017;91:e02199. https:/ / doi.org/ 10.1128/JVI.02199-16

5. Poen MJ, Venkatesh D, Bestebroer TM, Vuong O, Scheuer RD, Oude Munnink BB, et al. Co-circulation of genetically distinct highly pathogenic avian influenza A clade 2.3.4.4 (H5N6) viruses in wild waterfowl and poultry in Europe and East Asia, 2017-18. Virus Evol. 2019;5:vez004. https:/ /doi. org/10.1093/ve/vez004

6. Yu Z, Gao X, Wang T, Li Y, Li Y, Xu Y, et al. Fatal H5N6 avian influenza virus infection in a domestic cat and wild birds in China. Sci Rep. 2015;5:10704. https:/ / doi.org/ 10.1038/srep10704

7. Tsunekuni R, Yaguchi Y, Kashima Y, Yamashita K, Takemae N, Mine J, et al. Spatial transmission of H5N6 highly pathogenic avian influenza viruses among wild birds in Ibaraki Prefecture, Japan, 2016-2017. Arch Virol. 2018; 163:1195-207. https://doi.org/10.1007/s00705-018-3752-7

8. Hiono T, Okamatsu M, Matsuno K, Haga A, Iwata R, Nguyen LT, et al. Characterization of H5N6 highly pathogenic avian influenza viruses isolated from wild and captive birds in the winter season of 2016-2017 in Northern Japan. Microbiol Immunol. 2017;61:387-97. https:/ / doi.org/ 10.1111/1348-0421.12506

9. $\mathrm{Li} \mathrm{C}, \mathrm{Bu} \mathrm{Z}, \mathrm{Chen} \mathrm{H}$. Avian influenza vaccines against H5N1 'bird flu'. Trends Biotechnol. 2014;32:147-56. https://doi.org/10.1016/j.tibtech.2014.01.001

10. Zeng X, Chen X, Ma S, Wu J, Bao H, Pan S, et al. Protective efficacy of an $\mathrm{H} 5 / \mathrm{H} 7$ trivalent inactivated vaccine produced from Re-11, Re-12, and H7-Re2 strains against challenge 
with different $\mathrm{H} 5$ and $\mathrm{H} 7$ viruses in chickens. J Integr Agric. 2020;19:2294-300. https://doi.org/10.1016/S20953119(20)63301-9

11. Shi J, Deng G, Ma S, Zeng X, Yin X, Li M, et al. Rapid evolution of H7N9 highly pathogenic viruses that emerged in China in 2017. Cell Host Microbe. 2018;24:558-568.e7. https://doi.org/10.1016/j.chom.2018.08.006

12. Zeng X, Tian G, Shi J, Deng G, Li C, Chen H. Vaccination of poultry successfully eliminated human infection with H7N9 virus in China. Sci China Life Sci. 2018;61:1465-73. https://doi.org/10.1007/s11427-018-9420-1

13. Suttie A, Deng YM, Greenhill AR, Dussart P, Horwood PF, Karlsson EA. Inventory of molecular markers affecting biological characteristics of avian influenza A viruses. Virus Genes. 2019;55:739-68. https:// doi.org/10.1007/s11262-019-01700-z
14. World Organisation for Animal Health. Avian influenza ((infection with avian influenza viruses). In: Manual of diagnostic tests and vaccines for terrestrial animals. 2019 [cited Mar 10]. https://www.oie.int/fileadmin/Home/eng/ Health_standards/tahm/3.03.04_AI.pdf

15. Chen H, Li Y, Li Z, Shi J, Shinya K, Deng G, et al. Properties and dissemination of $\mathrm{H} 5 \mathrm{~N} 1$ viruses isolated during an influenza outbreak in migratory waterfowl in western China. J Virol. 2006;80:5976-83. https:// doi.org/10.1128/JVI.00110-06

Address for correspondence: Hualan Chen, Harbin Veterinary Research Institute, Chinese Academy of Agricultural Sciences, No. 678 Haping Rd, Harbin, Heilongjiang, 150069, China; email: chenhualan@caas.cn

\section{October 2020}

\section{Bacterial Infections}

- Operating Protocols of a Community Treatment Center for Isolation of Patients with Coronavirus Disease, South Korea

- Community Treatment Centers for Isolation of Asymptomatic and Mildly Symptomatic Patients with Coronavirus Disease, South Korea

- Clinical Course of Asymptomatic and Mildly Symptomatic Patients with Coronavirus Disease Admitted to Community Treatment Centers

- Nationwide External Quality Assessment of SARS-CoV-2 Molecular Testing, South Korea

- Impact of Social Distancing Measures on Coronavirus Disease Healthcare Demand, Central Texas, USA

- Multicenter Prevalence Study Comparing Molecular and Toxin Assays for Clostridioides difficile Surveillance, Switzerland

- Effectiveness of 23-Valent Pneumococcal Polysaccharide Vaccine against Invasive Pneumococcal Disease in Adults, Japan, 2013-2017

- Sequential Acquisition of Human Papillomavirus Infection at Genital and Anal Sites, Liuzhou, China

- Association between Shiga ToxinProducing Escherichia coli O157:H7 stx Gene Subtype and Disease Severity, England, 2009-2019

- Rapid, Sensitive, Full-Genome Sequencing of Severe Acute Respiratory Syndrome Coronavirus 2

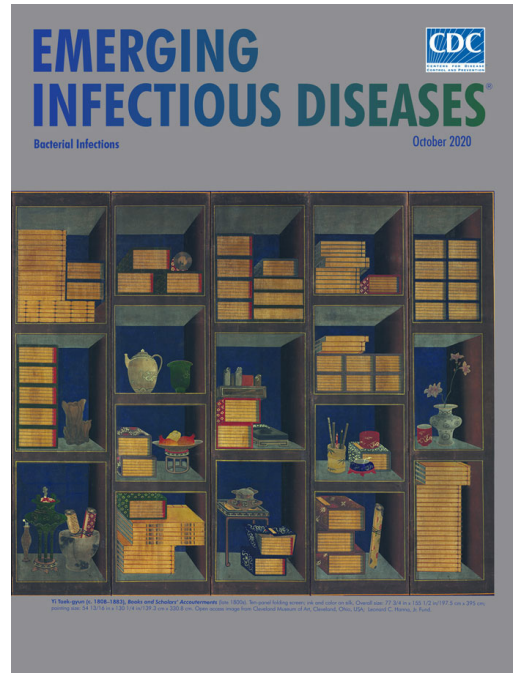

- Effect of Nonpharmaceutical Interventions on Transmission of Severe Acute Respiratory Syndrome Coronavirus 2, South Korea, 2020

- Main Routes of Entry and Genomic Diversity of SARS-CoV-2, Uganda

- High Proportion of Asymptomatic SARS-CoV-2 Infections in 9 Long-Term Care Facilities, Pasadena, California, USA, April 2020

- Tickborne Relapsing Fever, Jerusalem, Israel, 2004-2018

- Seawater-Associated Highly Pathogenic Francisella hispaniensis Infections Causing Multiple Organ Failure
- Basic Reproduction Number of Chikungunya Virus Transmitted by Aedes Mosquitoes

- Deaths Associated with Pneumonic Plague, 1946-2017

- Emerging Sand Fly-Borne Phlebovirus in China

- Drug Resistance Spread in 6 Metropolitan Regions, Germany, 2001-2018

- Human Adenovirus B7-Associated Urethritis after Suspected Sexual Transmission, Japan

- Polyester Vascular Graft Material and Risk for Intracavitary Thoracic Vascular Graft Infection

- Silent Circulation of Rift Valley Fever in Humans, Botswana, 2013-2014

- Limitations of Ribotyping as Genotyping Method for Corynebacterium ulcerans

- Seoul Orthohantavirus in Wild Black Rats, Senegal, 2012-2013

- Contact Tracing during Coronavirus Disease Outbreak, South Korea, 2020

- Pooling Upper Respiratory Specimens for Rapid Mass Screening of COVID-19 by Real-Time RT-PCR

- Coronavirus Disease among Persons with Sickle Cell Disease, United States, March 20-May 21, 2020

- Eliminating Spiked Bovine Spongiform Encephalopathy Agent Activity from Heparin

\section{EMERGING}

